\title{
Clásulas sociales en la contratación pública: nuevos instrumentos para el fomento del empleo a nivel local
}

\author{
Social clauses in public procurement: new tools to promote \\ local employment \\ Juan BERNETE GARCÍA \\ Observatorio Local de Empleo del Ayuntamiento de Fuenlabrada \\ jbernete@gmail.com
}

Recibido: 20/11/2012

Revisado: $03 / 13 / 2012$

Aceptado: $07 / 02 / 2013$

Disponible on line: 16/04/2013

\begin{abstract}
Resumen
En este artículo se aborda la incorporación de las cláusulas sociales en la contratación pública como nuevos instrumentos que posibilitan políticas públicas de fomento del empleo, la igualdad de oportunidades y la lucha contra la exclusión social, fundamentalmente a nivel local. Se explica cómo el marco jurídico actual contempla diversas modalidades de incorporación de criterios sociales y medioambientales en los pliegos de contratación, tanto en la fase de admisión como de valoración de las ofertas y las condiciones de ejecución. También se analizan diversas experiencias y buenas prácticas, tanto a nivel autonómico como en el ámbito local, con el objeto de explicar y demostrar que la aplicación de dichas cláusulas es plenamente legal, si se emplean correctamente, y se trata únicamente de voluntad política el llevarlas a cabo por una Administración Pública. Una de las conclusiones principales es que se trata de una herramienta innovadora que puede dar un nuevo impulso a las políticas de empleo y de inclusión social, a la inserción laboral de colectivos excluidos del mercado de trabajo como parte de las políticas sociales de lucha contra la exclusión.
\end{abstract}

Palabras clave: cláusulas sociales, contratación pública, empleo, inserción sociolaboral, mercados sociales.

\begin{abstract}
With this article we address the issue of the incorporation of social clauses in public procurement as new tools that enable public policies to promote employment, equal opportunities and the fight against social exclusion, mainly locally. It explains how the current legal framework contemplates incorporating various forms of social and environmental criteria in procurement specifications, both at the stage of admission, as assessment of tenders and execution conditions. It also discusses various experiences and good practices at regional level and at the local level, in order to explain and demonstrate the use of social clauses is fully legal, if properly applied, its only political will to implement them in public administration.

One of the main conclusions is that it is an innovative tool that can give new impetus to employment policies and social inclusion, employability of groups excluded from the labor market as part of social policies to combat exclusion. Keywords: social clauses, public procurement employment, labor integration, social markets
\end{abstract}

Referencia normalizada: Bernete García, J. (2013): «Cláusulas sociales en la contratación pública: nuevos instrumentos para el fomento del empleo a nivel local». Cuadernos de Trabajo Social, 26(1): 85-94.

Sumario: Introducción. 1. Cláusulas sociales y políticas de empleo e inclusión social. 2. Modalidades de cláusulas sociales en la normativa. 3. Ejemplos y buenas prácticas de implantación de cláusulas sociales. 4. Conclusiones. 5. Referencias bibliográficas.

\section{Introducción}

La contratación pública se basa actualmente en unos criterios objetivos (condiciones técnicas y precio) y la aplicación de unos principios (libre concurrencia y publicidad), no teniendo en cuenta otros principios más importantes de carácter ético, social y solidario, como el fomento del empleo de personas y colectivos desfavorecidos, la igualdad de oportunidades entre mujeres y hombres, el empleo de calidad y con derechos, la protección medioambiental, el comercio justo, en definitiva la compra ética y responsable. 
En el siglo XIX la subasta y el precio eran los únicos sistemas de contratación pública. En el siglo XX surge el concurso, incorporando otros criterios de adjudicación además del precio; pero es en el presente siglo XXI cuando empiezan a incorporarse los criterios sociales para valorar las mejores ofertas. El punto de inflexión, en mi opinión, se dio con la sentencia del Tribunal Supremo (23 de mayo de 1997) en la que se expresa: «La administración no está obligada a aceptar el mejor precio, sino la oferta más favorable al interés público».

En el marco normativo europeo la Directiva 2004/18/CE es la que ha asumido y regulado expresamente la introducción de criterios sociales en la contratación pública.

\section{Cláusulas sociales y políticas de empleo e inclusión social}

El peso de los contratos públicos en nuestro país oscila entre el 11 por ciento y el 13 por ciento del PIB (en la Unión Europea supone entre el 15 por ciento y el 17 por ciento del PIB). La Administración Pública se convierte en agente económico de primer orden y cabe exigirle eficacia y eficiencia en el gasto público y su responsabilidad social, puesto que ésta: a) es gestora de fondos y presupuestos públicos y debe impulsar efectos sociales y de empleo; b) es consumidora, debe promover un fin ético en sus compras; c) ha de actuar como referente para el resto de agentes sociales y económicos llamados a ejercer su responsabilidad social corporativa (RSC).

Las medidas de las políticas de empleo y de inclusión social en los últimos años no se renuevan y siguen apuntando únicamente a mejorar la «empleabilidad» y a remover ciertos obstáculos de acceso al mercado de trabajo, mediante incentivos a la contratación; pero no se cuestionan el modelo de crecimiento económico como principal factor causante de la exclusión. Seguimos teniendo mercados de trabajo excluyentes y discriminadores.

En este escenario de crisis y recortes, las políticas sociales son insuficientes: el gasto social en España se sitúa 7 puntos por debajo de la Unión Europea. Facilitar el empleo de los sectores de la población más vulnerables es un deber constitucionalmente establecido.
Las cláusulas sociales son, probablemente, el instrumento más eficaz con el que cuenta hoy en día la Administración para fomentar empleo. La Comisión Europea (2010) define ampliamente el concepto de contrataciones públicas socialmente responsables:

Las «CPSR» se refieren a las operaciones de contratación que tienen en cuenta uno o más de los siguientes aspectos sociales: oportunidades de empleo, trabajo digno, cumplimiento con los derechos sociales y laborales, inclusión social (incluidas las personas con discapacidad), igualdad de oportunidades, diseño de accesibilidad para todos, consideración de los criterios de sostenibilidad, incluidas las cuestiones de comercio ético y un cumplimiento voluntario más amplio de la responsabilidad social de las empresas (RSE), a la vez que se respetan los principios consagrados en el Tratado de la Unión Europea (TUE) y las Directivas de contratación.

Se trata de concebir la contratación administrativa como un instrumento que posibilite políticas públicas transversales. Es una herramienta innovadora que puede dar un nuevo impulso a las políticas de empleo, a la inserción laboral de colectivos excluidos del mercado de trabajo, como parte de las políticas sociales de lucha contra la exclusión. Conecta perfectamente con uno de los ejes de la Estrategia Europea de «Inclusión Activa» que hace referencia a la vinculación con medidas activas del mercado laboral propiciando mercados laborales inclusivos.

Ya se decía en el Informe del Congreso de los Diputados para promover la Responsabilidad Social de las Empresas ${ }^{1}$ que:

La compra y contratación públicas deben ser claras herramientas para el impulso de la RSE, enviando señales al mercado de que serán reconocidas y premiadas las empresas que brindan a la sociedad un valor adicional en materia ambiental y/o social. Claros ejemplos de esto pueden ser la inclusión de cláusulas sociales que permitan discriminar positivamente a favor de las empresas de inserción o de los productos de comercio justo.

Mediante la aplicación de las cláusulas propuestas se consigue además de la realización de las obras o prestación de servicios, una ren-

1 Informe de la Subcomisión Parlamentaria sobre la responsabilidad social de las empresas en 2006, aprobado posteriormente por el Consejo de Ministros el 30 de enero de 2009. 
tabilidad social que supone una inversión que contribuye, por un lado, a disminuir el gasto público destinado a estos fines (programas sociales, rentas básicas, subsidios por desempleo o gastos sanitarios) y, por otro lado, a generar ingresos a la Hacienda Pública (IRPF, IVA y Seguridad Social).

Permiten establecer una nueva relación entre la Administración y las empresas, para que éstas asuman su compromiso con la responsabilidad social corporativa, integrando las preocupaciones sociales y ecológicas en sus actividades comerciales e implicándolas en la consecución de objetivos sociales.

Exceptuando casos ejemplarizantes existentes, las administraciones públicas no han tomado conciencia de su capacidad y su potencial en lograr objetivos sociales a través de la contratación. Es necesario, por lo tanto, seguir insistiendo para que, al adjudicar sus contratos, las administraciones públicas dejen de aplicar el precio (la mejor oferta económica) de forma preferente y adopten una postura más responsable que favorezca la inserción sociolaboral, el comercio justo o el empleo de calidad en sus compras públicas.

\section{Modalidades de cláusulas sociales en la normativa}

El impulso a la incorporación de cláusulas o criterios sociales en la contratación pública vino en nuestro país de la mano de la Ley 30/2007, de Contratos del Sector Público, que ofrecía un margen para implementar dichos criterios en los pliegos de contrataciones (esta ley ha estado en vigor hasta finales de 2011). En la actualidad es el Real Decreto-Ley 3/2011, Texto Refundido de la Ley de Contratos del Sector Público, el que da continuidad a la Ley de Contratos del Sector Público. Así, con la normativa en vigor se puede incorporar diversas modalidades de cláusulas sociales en los contratos públicos:

\section{Reservas de contratos. Disposición Adicio- nal $5^{\mathrm{a}}$ RDL 3/2011}

Una de las fórmulas que la legislación habilita para limitar la admisión de licitadores a determinadas empresas, consiste en aplicar esta Disposición Adicional sobre contratos reservados.

Podrá reservarse la participación en los procedimientos de adjudicación de contratos a Centros
Especiales de Empleo, o reservar su ejecución en el marco de programas de empleo protegido, cuando al menos el 70 por 100 de los trabajadores afectados sean personas con discapacidad.

Permite adjudicar contratos directamente a los centros especiales de empleo y se aplica por analogía a las empresas de inserción. Se trata de establecer unos porcentajes mínimos que no distorsionan la libre concurrencia y fomentan mercados inclusivos en el territorio.

La implantación de cláusulas sociales, y especialmente la de reservas de mercados a determinadas empresas sociales supone la idea de la discriminación positiva. La jurisprudencia del Tribunal Constitucional reconoce esta figura ampliamente: «El principio de igualdad permite el tratamiento desigual ante situaciones de hecho desiguales» (STC 128/1987 de 16 de julio) o también:

La actuación de los poderes públicos para remediar, así, la situación de determinados grupos sociales definidos y colocados en posiciones de innegable desventaja en el ámbito laboral, por razones que resultan de tradiciones y hábitos profundamente arraigados en la sociedad y difícilmente eliminables, no puede considerarse vulneradora del principio de igualdad, aún cuando se establezca para ellos un trato más favorable, pues se trata de dar tratamiento distinto a situaciones efectivamente distintas (STC 128/1987 de 16 de julio).

El Comité Económico y Social Europeo (CE$\mathrm{SE})$ recomienda la adjudicación obligatoria de un número o porcentaje determinado de contratos específicos a Centros Especiales de Empleo en aquellos Estados miembros en los que exista un número importante de personas con discapacidad que pueden trabajar pero que siguen inactivas, como ocurre en España ( Cabra de Luna, 2012; Dictamen 2012/C 191/16).

\section{Criterios de valoración de las ofertas. Artí- culo 150. RDL 3/2011}

Para la valoración de las proposiciones y la determinación de la oferta económicamente más ventajosa deberá atenderse a criterios directamente vinculados al objeto del contrato, tales como la calidad, el precio (...) las características medioambientales o vinculadas con la satisfacción de exigencias sociales que respondan a necesidades, definidas en las especificaciones del contrato, propias de las catego- 
rías de población especialmente desfavorecidas a las que pertenezcan los usuarios o beneficiarios de las prestaciones a contratar.

En la fase de valoración, las cláusulas sociales a incorporar deben atenerse a algunos criterios, ya que de lo contrario podrían ser objeto de impugnaciones o anulaciones: 1. Que estén directamente vinculados al objeto del contrato. 2. Que sean objetivos, públicos, detallados y cuantificables. 3. Que tengan una ponderación moderada, carácter complementario de los restantes criterios. Por ejemplo que un porcentaje de la plantilla que ejecutará el contrato sean personas con dificultades de acceso al empleo.

\section{Disposición Adicional 4ª . RDL 3/2011}

Mediante esta cláusula se puede establecer una preferencia en la adjudicación de contratos a determinadas empresas $y$ entidades sociales:

- Empresas que tengan en su plantilla un número de trabajadores con discapacidad superior al 2 por 100 .

- Empresas de inserción, con un 30 por ciento de sus puestos de trabajo con personas pertenecientes a los colectivos en situación de exclusión.

- Entidades sin ánimo de lucro, siempre que su finalidad tenga relación directa con el objeto del contrato.

- Organizaciones de Comercio Justo, en contratos que tengan como objeto productos en los que exista alternativa de Comercio Justo.

«Siempre que dichas proposiciones igualen en sus términos a las más ventajosas», lo que resulta una mera declaración de principios, ya que en la práctica no suelen darse casos de empate. No obstante, está bien incorporarlo en los pliegos ya que ayuda a sensibilizar sobre la economía social y solidaria.

Además de la preferencia en la contratación, la Disposición Adicional Cuarta establece que:

Los órganos de contratación ponderarán en los supuestos que ello sea obligatorio, que los licitadores cumplen lo dispuesto en la Ley 13/1982, de 7 de abril, de integración social de los minusválidos, relativo a la obligación de contar con un dos por ciento de trabajadores con discapacidad o adoptar las medidas alternativas correspondientes.
Condiciones especiales de ejecución del contrato. Artículo 118. RDL 3/2011

Los órganos de contratación podrán establecer condiciones especiales en relación con la ejecución del contrato, siempre que sean compatibles con el derecho comunitario y se indiquen en el anuncio de licitación y en el pliego o en el contrato. Estas condiciones de ejecución podrán referirse, en especial, a consideraciones de tipo medioambiental o a consideraciones de tipo social, con el fin de promover el empleo de personas con dificultades particulares de inserción en el mercado laboral, eliminar las desigualdades entre el hombre y la mujer en dicho mercado, combatir el paro, favorecer la formación en el lugar de trabajo, u otras finalidades que se establezcan con referencia a la estrategia coordinada para el empleo, o garantizar el respeto a los derechos laborales básicos a lo largo de la cadena de producción mediante la exigencia del cumplimiento de las Convenciones fundamentales de la Organización Internacional del Trabajo.

Es, sin duda, el más relevante de la Ley para posibilitar, de una manera más efectiva, la implantación de cláusulas sociales. Aquí la cláusula forma parte del contrato y es una obligación inherente al mismo que los licitadores por el hecho de presentar su oferta se comprometen a cumplir en fase de ejecución. Su incumplimiento pudiera dar lugar a la rescisión del contrato. Por ejemplo que la empresa adjudicataria deba ineludiblemente contratar a un número de personas desempleadas de colectivos con mayores dificultades en el mercado de trabajo.

\section{Otras modalidades de cláusulas sociales}

Pueden incorporarse otras variantes de cláusulas sociales, tales como:

- Con carácter previo, en la definición del objeto del contrato, que es potestad del organismo contratante. Así, por ejemplo, en lugar de que el objeto del contrato sea «el mantenimiento de zonas verdes», se podría expresar como «proyecto de empleo para personas con discapacidad, mediante la actividad de mantenimiento de zonas verdes.»

- Como criterio de admisión, en la fase de selección, exigiendo requisitos previos de carácter social o la acreditación de experiencia o solvencia en materia de inserción sociolabo- 
ral para que la empresa pueda ser contratada. Lo que establece la Comisión Europea (2001) es que las exigencias sobre solvencia técnica y profesional deben estar relacionadas con el objeto del contrato.

La exigencia es lógica: si para construir una central térmica se requiere acreditar una experiencia concreta y un conocimiento en ese tipo de tecnología, si el objeto del contrato y las especificaciones técnicas contemplan la contratación de colectivos desfavorecidos del mercado laboral, deberá requerirse experiencia específica en inserción laboral para prestar debidamente el contrato. Por ejemplo, exigir que se trate de un Centro Especial de Empleo, una Empresa de Inserción o Entidades de Acción Social dedicadas a inserción laboral de colectivos desfavorecidos.

Las cláusulas sociales pueden implantarse indistintamente en los procedimientos contemplados en la ley de contratos: abierto, restringido, negociado con y sin publicidad, incluso se pueden utilizar los contratos menores.

\section{Ejemplos y buenas prácticas de implanta- ción de cláusulas sociales}

\section{A nivel autonómico}

Cataluña es una de las administraciones pioneras en reservar mercados a empresas sociales. Mediante estas reservas sociales, desde el año 2004, con un importe anual superior a los 7.000.000 euros generan un volumen muy importante de trabajo a estas empresas sociales, generando a su vez un número considerable de empleos de inserción en el territorio. Por ejemplo, solo entre las empresas de inserción se generan unos 300 empleos al año para personas en situación de exclusión social (Lesmes Zabalegui, 2006).

Reproducimos aquí parte del articulado de la Ley 7/2004, de la Comunidad Autónoma de Cataluña, de medidas fiscales y administrativas, artículo 35 (Fomento de objetivos sociales en la contratación):

1. Todos los departamentos, organismos autónomos y empresas públicas de la Generalitat deben reservar determinados contratos administrativos de obras, suministros o servicios a centros de inserción laboral de discapacitados, empresas de inserción sociolaboral y enti- dades sin ánimo de lucro dedicadas a la inserción laboral de colectivos desfavorecidos.

2. Los objetos contractuales susceptibles de reserva son las obras y servicios de conservación y mantenimiento de bienes inmuebles; los servicios de mensajería, correspondencia y distribución, de artes gráficas, de limpieza y lavandería, de restauración y de recogida y transporte de residuos, y los servicios y suministros auxiliares para el funcionamiento de la Administración.

3. La reserva se fija entre el 10 por ciento y el 20 por ciento del importe presupuestario adjudicado exclusivamente mediante contrato menor o por procedimiento negociado por razón de la cuantía económica.

4. La Administración local puede aplicar la reserva social en los términos que establezca el acuerdo correspondiente del pleno de la corporación.

Asimismo, como ejemplo de buena práctica de aplicación de cláusulas de condiciones especiales de ejecución, el Programa Posa't a Punt (Puesta a Punto) de la Generalitat de Cataluña es una innovadora y pionera iniciativa (desde 1997) en las licitaciones públicas de obras de rehabilitación y mantenimiento de vivienda pública e incorpora como exigencia de ejecución un compromiso de reserva de puestos de trabajo de inserción, como proyecto de mejoras al pliego.

Este programa ha permitido que empresas de economía social y de inserción concursen en contratos de obras por sí solas o en unión temporal de empresas (UTE) con grandes empresas. La empresa adjudicataria debe presentar un proyecto de inserción sociolaboral y reservar un número de puestos de trabajo para trabajadores que provengan de dispositivos locales de empleo e inserción y realizar un itinerario de inserción individual. El coste de los tutores de inserción corre a cargo de las empresas adjudicatarias de las obras. Hasta el año 2005 se habían generado más de 1.300 puestos de trabajo, ocupados por personas con dificultades de acceso al empleo (Lesmes $\mathrm{Za}$ balegui, 2006).

Este mismo modelo de Posa't a Punt se ha utilizado también en Andalucía, en la Empresa Pública de Suelo, para la construcción de viviendas de protección oficial (VPO). 
La Comunidad Foral de Navarra también dispone, desde el año 2006, de su propia normativa sobre contratos públicos, la Ley Foral 6/2006, de 9 de junio, de contratos públicos de Navarra en cuyo preámbulo se da de las cláusulas sociales «una visión instrumental al servicio de valores superiores de las políticas públicas frente a la visión economicista y simplista». En un primer momento, la reserva de mercado para centros especiales de empleo y empresas de inserción era potestativa de los distintos departamentos públicos y no establecía un porcentaje obligatorio, si bien se establecía el 20 por ciento como tope máximo. Posteriormente se estableció un porcentaje obligatorio del 6 por ciento. Aún así el grado de cumplimiento era mínimo y sólo cuando las organizaciones del sector presionaron se creó una comisión de apoyo al empleo protegido que empieza a dar sus frutos ahora. En el año 2012 habrá reservado un porcentaje en torno al 3 por ciento del total de sus contratos públicos, con un importe aproximado de 4 millones de euros.

En el año 2008, el Consejo de Gobierno del País Vasco aprobó un acuerdo, Resolución 6/2008, de 2 de Junio, del Acuerdo del Consejo de Gobierno, para incorporar cláusulas sociales en la contratación pública. El antecedente inmediato fue la aprobación, en junio de 2007, de una proposición no de ley sobre inclusión de cláusulas sociales, que comportaba un mandato para el Gobierno Vasco de elaborar e impulsar dicho acuerdo. En ese acuerdo se recogen diversas modalidades de cláusulas sociales que se deben incorporar de forma taxativa en los pliegos de todos los departamentos, organismos autónomos y empresas públicas del Gobierno vasco, siendo referencia también para las administraciones locales. Cabe destacar las siguientes medidas:

- Reserva de contratos a centros especiales de empleo y a empresas de inserción sociolaboral: Todas las entidades pertenecientes al sector público de la Comunidad Autónoma deben reservar anualmente para ese fin un importe económico que fijará el Departamento de Hacienda o los departamentos competentes en materia de inserción laboral, previa consulta con las asociaciones empresariales representativas. En el caso de las empresas de inserción, los contratos que pueden adjudicarse son los denominados menores (50.000 euros para obras y 18.000 euros para servicios y suministros) y procedimientos negociados $(1.000 .000$ euros en obras, 500.000 en gestión de servicios públicos y 100.000 euros para suministros y servicios).

- Obligación a todas las empresas adjudicatarias y en todos los contratos públicos del compromiso de incorporar en la ejecución de la prestación contratada al menos un 20 por ciento de personas desempleadas que se encuentren en especial dificultad para acceder al empleo. Se establecen, no obstante, algunas excepciones, como que la empresa adjudicataria no precise nuevas contrataciones de personal o en casos de subrogación de la plantilla. Además se establecen medidas alternativas, como la obligación de la empresa adjudicataria de subcontratar el 5 por ciento del importe con empresas inscritas en el Registro de Centros Especiales de Empleo o de Empresas de Inserción.

Sin duda, las organizaciones representativas del sector han sido decisivas en este proceso de presión y sensibilización para que los representantes políticos en el Parlamento adopten estas medidas que benefician a todos y no perjudican a nadie, o sea, que todos ganan.

\section{A nivel local}

Muchos municipios llevan años trabajando con cláusulas sociales, otros están incorporándolas en los pliegos o bien haciendo declaraciones de intenciones en los planes municipales o en los plenos asumiendo el compromiso de implantarlas progresivamente en los pliegos de contratación. Citaremos algunas experiencias, a modo de ejemplos de buenas prácticas, lo que no debe interpretarse que son las mejores ni las únicas.

El Ayuntamiento de Barcelona: en diciembre de 2008 se adoptó el Acuerdo de gobierno para la contratación responsable: nuevo impulso de la contratación social y ambiental. Se incorpora, por un lado, la reserva social, a modo de reserva de determinados contratos de obras, suministros y servicios a determinadas empresas sociales, como centros especiales de empleo y empresas de inserción en procedimiento negociado sin publicidad y contratos menores. El importe anual de dicha reserva no puede ser inferior a 5.000.000 euros ni supe- 
rior al 20 por ciento del total de la contratación por dichos procedimientos. También se incorporan criterios sociales para impulsar la compra verde y la creación de un portal web de compra social integrado en la web municipal.

En 2011 se ha incorporado nuevas cláusulas sociales en los pliegos de contratación de mantenimiento de edificios y vías públicas, para potenciar la inserción laboral de colectivos desfavorecidos y fomentar un mercado social a empresas sociales. Los licitadores se tienen que comprometer, de forma obligatoria, para la ejecución del contrato a contratar unos mínimos (en función de los importes de los contratos adjudicados) de personas de colectivos desfavorecidos, en colaboración con el servicio municipal de empleo y desarrollo local y compromiso de subcontratar un mínimo del 5 por ciento del presupuesto de ejecución material de obra con centros especiales de empleo y empresas de inserción.

El Ayuntamiento de Girona: en el año 1.999 acuerda una cláusula social en una parte de los contratos de obras superiores a 150.000 euros, por la que se establece que un porcentaje a concretar de los trabajadores que formen parte de la plantilla que ejecute el contrato, sean elegidos por la empresa adjudicataria entre aquellos que forman parte del programa de incorporación sociolaboral municipal.

El Ayuntamiento de Sevilla aprobó ${ }^{2}$ igualmente en 2006 unos pliegos de cláusulas administrativas generales de calidad social en la contratación pública, en todas las contrataciones de obras y servicios igual o superior a 150.000 euros y plazo de ejecución superior a nueve meses. En dichas contrataciones, las empresas licitadoras están obligadas a:

- Incorporar en la obra o servicio adjudicado al menos un 10 por ciento de personas provenientes de los colectivos de personas desempleadas con dificultad para acceder al empleo.

- Toda empresa adjudicataria que cuente con una representación de mujeres en plantilla desequilibrada, deberá realizar durante la ejecución del contrato al menos una nueva contratación de mujer o transformar al menos una contratación temporal de mujer en contratación indefinida.
- Durante la ejecución del contrato la empresa deberá cumplir y acreditar que al menos el 30 por ciento de la plantilla adscrita al servicio objeto del contrato es indefinida.

- Durante la ejecución del contrato deberán realizar acciones de sensibilización, formación y orientación dentro de la jornada laboral, para el conocimiento de los derechos establecidos en la ley o en el convenio colectivo de aplicación, en materia de conciliación de la vida laboral y familiar de las personas trabajadoras.

El Ayuntamiento de Avilés: aprobó en el año 2009 la Instrucción para la incorporación de cláusulas sociales en los contratos públicos del Ayuntamiento de Avilés (B.O.P.V., 273, 2009). Utiliza un amplio abanico de posibilidades de introducir criterios sociales, de acuerdo con la legislación: contratos reservados a empresas sociales; criterios sociales de valoración para la adjudicación; solvencia técnica; objeto del contrato y condiciones de ejecución. Aborda diferentes aspectos sociales: inserción sociolaboral, discapacidad, seguridad y salud laboral, igualdad de oportunidades entre mujeres y hombres, y calidad en el empleo. Contiene además un criterio de adjudicación relativo al «Proyecto de Inserción Sociolaboral» cuando éste forme parte fundamental del objeto del contrato. Se refieren con detalle las «Condiciones especiales de ejecución de carácter social del Pliego de Cláusulas Administrativas Particulares incorporando medidas de inserción sociolaboral o de creación de empleo para personas con dificultades de acceso al mercado laboral».

Fue elegida como la mejor práctica nacional y una de las mejores prácticas europeas en la Conferencia Europea sobre Responsabilidad Social de las Empresas (marzo de 2010, Palma de Mallorca), siendo seleccionada como modelo de fomento de la RSE desde las Administraciones Públicas.

\section{Otras experiencias municipales}

El Ayuntamiento de Getxo: es pionero en incorporar cláusulas sociales a través del objeto del contrato. Así, por ejemplo contrata «Las obras de rehabilitación de una vivienda municipal, mediante la realización de procesos de

${ }^{2}$ La Junta de Gobierno de la Ciudad de Sevilla aprobó el 4 de abril de 2007. Recibió el dictamen favorable del Consejo Consultivo de Andalucía con fecha 16 de mayo de 2007. 
inserción sociolaboral a través del empleo, la formación y el acompañamiento». $O$ este otro: «El contrato que sobre la base del presente pliego se realice tendrá por objeto la ocupación de personas desempleadas pertenecientes a colectivos con dificultades para el acceso al mundo laboral o que se encuentren en situación de riesgo de exclusión, para la prestación del servicio de mensajería», lo que le faculta en mejor medida para valorar el proyecto de inserción laboral que presenta la empresa licitadora para la adjudicación del contrato.

El Ayuntamiento de Amurrio: La empresa adjudicataria del servicio de gestión del teléfono de atención ciudadana deberá garantizar obligatoriamente que entre un 40 por ciento- 75 por ciento de la plantilla se cubrirá con trabajadores en situación o riesgo de exclusión social, derivados a través de los Servicios Sociales de Base del entorno, y realizando los itinerarios de inserción individualizados de formación y el acompañamiento para la incorporación al mercado ordinario o iniciativas de auto-ocupación.

El Ayuntamiento de Salamanca: al igual que en el caso de Getxo, contratación del desarrollo de un proyecto de inserción sociolaboral con personas en situación o riesgo de exclusión social mediante la realización de trabajos de conservación, mantenimiento y mejora de determinadas zonas ajardinadas; así como para un contrato de trabajos de limpieza mediante un proyecto de inserción sociolaboral.

El Ayuntamiento de Huelva: aprobó su pliego de cláusulas administrativas generales de calidad social en la contratación pública, que vinculan a todas las licitaciones, como parte de su Plan de fomento de la calidad en el empleo.

El Ayuntamiento de A Coruña: Ha incluido en los pliegos de los proyectos que se ejecuten en la ciudad (superiores a 120.000 euros) una serie de cláusulas sociales con el fin de favorecer la contratación de personas en situación de riesgo. Se pretende que las empresas incluyan en sus propuestas medidas que apoyen la integración laboral de dichos colectivos, así como la conciliación de la vida laboral y familiar.

El Ayuntamiento de Burgos: para la contratación de servicios de atención domiciliaria establece cláusulas sociales del tipo: «Se valorará la contratación por el licitador de personas pertenecientes a los colectivos con mayores dificultades de inserción, en especial los definidos como preferentes de actuación en los Planes Nacionales de Acción para el Empleo (...) y que intervengan directamente en la prestación de los servicios del Lote 2 (Comida y lavandería a domicilio) y 3 (Respiro familiar) que son objeto del concurso»)(Concurso para la contratación de la gestión de servicios de atención domiciliaria del Ayuntamiento de Burgos, 2005).

En Sant Boi de Llobregat se crea en el año 2000 un servicio municipal de inserción con fórmula de empresa de inserción de titularidad municipal (Corinsert) para desarrollar servicios públicos municipales, como la jardinería, construcción y actuaciones medioambientales.

El Ayuntamiento de Bilbao: aprobó la modificación de los pliegos tipo de cláusulas administrativas particulares, en la que a aquellas empresas que contratasen a desempleados o desempleadas con especiales dificultades de acceso al mercado de trabajo laboral le corresponderá con porcentajes de puntuación entre 10 por ciento y 15 por ciento, cuando al menos el 20 por ciento de la plantilla pertenezca a los colectivos especificados.

El Ayuntamiento de Alcázar de San Juan: incorpora cláusulas sociales en el concurso para la adjudicación de suelo industrial, primando la implantación de empresas socialmente responsables y la creación de empleo en la localidad, incorporando trabajadores y trabajadoras con especiales dificultades de acceso al mercado de trabajo, compromisos de llevar a cabo programas de inserción sociolaboral o contratar con empresas cuyo objetivo principal sea la contratación de estos colectivos.

Otros entidades locales que están incorporando diversas modalidades de cláusulas sociales: Burlada, Castellón, Ceuta, Cobeña, Diputación Provincial de Málaga, Gijón, Hondarribia, Irún, Jaén, Las Gavias, Mancomunidad de servicios de la comarca de Pamplona, Manresa, Ondarroa, Palma de Mallorca, Puerto Real, Sabadell, Valdepeñas, Zaragoza, etc.

En el año 2009 se creó un grupo de trabajo, en el marco del Observatorio de la Exclusión Social y los Procesos de Inclusión en la Comunidad de Madrid, para analizar experiencias de cláusulas sociales en la contratación pública y elaborar propuestas a la Comunidad de Madrid. 
Participaron varias entidades como Asociación Madrileña de Empresas de Inserción (AMEI), Asociación FEAPS para el Empleo de las personas con discapacidad intelectual de Madrid (AFEM), Ideas-Comercio Justo, Fundación Mujeres y REAS Madrid (http://observatorioinclusion.wordpress.com/publicaciones).

\section{Conclusiones}

Mediante este uso inteligente de la contratación pública pueden darse nuevos impulsos a las políticas activas de empleo e inclusión sociolaboral, que tan drásticamente se están eliminando en el actual contexto político, dotando de mayor eficacia y sentido a los programas de formación, orientación e intermediación laboral en los ayuntamientos.

El volumen de contrataciones públicas tiene un peso presupuestario muy superior al de las partidas que cualquier administración dedique a políticas activas de empleo. Parece, por tanto, pertinente destinar parte de este presupuesto de contrataciones a fomentar el acceso a las mismas a colectivos de difícil acceso al mercado laboral, de forma complementaria a otras actuaciones.

Con el marco jurídico actual, las disquisiciones sobre la legalidad de las cláusulas sociales es un asunto del pasado. Las rigideces en la interpretación por parte de algunos organismos, cuando no de oposición, carecen ya de sentido y es necesario avanzar de modo progresivo para que nuestras administraciones pú- blicas utilicen sus contratos como eficaces instrumentos de lucha contra la exclusión social.

Conviene comenzar por una implantación progresiva, con experiencias piloto que permitan evaluar resultados. Antes de su implantación hay que prever la formación del personal técnico de secretaría e intervención para evitar posibles obstáculos. Resulta muy conveniente buscar el máximo apoyo político, que se puede concretar en un acuerdo de pleno, manifestando la voluntad de incorporar cláusulas sociales en los procesos de contratación pública. Igual de importante es llevar a cabo una labor de evaluación y seguimiento de la implantación de las cláusulas sociales, mostrando públicamente el impacto que dichas medidas han tenido sobre el empleo y la inserción en el territorio.

Es cierto que pueden suponer un incremento de la carga de trabajo en los departamentos de contratación. Hace falta liderazgo y voluntad política para conseguir que los equipos de trabajo tengan los recursos necesarios para poder llevar a cabo la implantación de estas medidas de contratación responsable.

Ya decía Pérez Llorca (2000) que: «Definir políticas de este tipo desde las administraciones públicas requieren del gestor un gran esfuerzo para alejarse de la rutina, y para no claudicar apriorísticamente, aceptando que los «procesos son lo que son», confundiendo muchas veces control de la legalidad con seguidismo de lo que siempre se ha hecho».

Otra forma de contratación pública es posible.

\section{Referencias bibliográficas}

Alves Bayern, M. (2012). El papel de las administraciones públicas en el fomento de las empresas de inserción. Cuadernos de Trabajo Social, 25 (1), 173-192.

Bernete García, J. (coord.) (2010). Hacia una contratación responsable. Propuestas para la incorporación de criterios sociales en la Comunidad de Madrid. Madrid: Observatorio de la Exclusión Social y los Procesos de Inclusión en la Comunidad de Madrid.

Comité Económico y Social Europeo. (2012). Dictamen INT/570-Mercado Europeo de los Contratos Públicos, relativo al «Libro Verde sobre la modernización de la política de contratación pública de la UE, hacia un mercado europeo de la contratación pública más eficiente». COM(2011) 897 final-2011/0437 (COD)](2012/C 191/16. Bruselas: Presidencia del Comité Económico y Social.

Comisión Europea. (2010). Adquisiciones sociales. Una guía para considerar aspectos sociales en las contrataciones públicas. Luxemburgo: Dirección General de Empleo, Asuntos Sociales e Igualdad de Oportunidades.

Comisión de las Comunidades Europeas. (15 de octubre de 2001). Comunicación interpretativa de la Comisión sobre la legislación comunitaria de contratos públicos y las posibilidades de integrar aspectos sociales en dichos contratos. COM(2001) 566 final. Recuperado de http://eur-lex.europa.eu/LexUriServ/LexUriServ.do?uri=COM:2001:0566:FIN:ES:PDF). 
Parlamento Europeo y Consejo de la Unión Europea. (marzo de 2004). Directiva 2004/17/CE del Parlamento Europeo y del Consejo sobre procedimientos de adjudicación de contratos públicos (L 134/113). Bruselas: Parlamento Europeo y Consejo.

Lesmes Zabalegui, S. (2006) Cláusulas sociales. Contratación Pública e inserción social. Bilbao: Ed. Proyecto LAMEGI (I. Comunitaria EQUAL).

Ley 13/1982, de 7 de abril, de integración social de los minusválidos. Boletín Oficial del Estado, 103, de 30 de abril de 1982.

Ley $3 / 2007$, de 22 de marzo, para la igualdad efectiva de hombres y mujeres. Boletín Oficial del Estado 71, de 23 de marzo de 2007.

Ley 30/2007, de 30 de octubre, de Contratos del Sector Público. Boletín Oficial del Estado, 261, de 31 de octubre de 2007.

Ley 44/2007, de 23 de diciembre, para la regulación del régimen de las empresas de inserción. Boletín Oficial del Estado, 229, de 14 de diciembre de 2007.

Ley 7/2004, de medidas fiscales y administrativas, de 16 de julio, de Cataluña. D.O.G.C. 4179 de 21 de julio de 2006, y Boletín Oficial del Estado, 235 de 29 de septiembre de 2006).

Ley Foral 6/2006, de 9 de junio, de contratos públicos de Navarra. Boletón Oficial de Navarra, 72 de 16 de junio de 2006 y Boletín Oficial del Estado, 158 de 4 de julio de 2006).

Pérez Llorca, J. et al (1999-2000). Las Empresas de Inserción a debate. Documentación Social [Monografía].

Real Decreto-Ley 3/2011, de 14 de noviembre, por el que se aprueba el Texto Refundido de la Ley de Contratos del Sector Público. Boletín Oficial del Estado, 276, de 16 de noviembre de 2011.

Resolución 6/2008, de 2 de junio, del Director de la Secretaría del Gobierno y de Relaciones con el Parlamento del País Vasco, por la que se dispone la publicación del Acuerdo adoptado por el Consejo de Gobierno «sobre incorporación de criterios sociales, ambientales y otras políticas públicas en la contratación de la Administración de la Comunidad Autónoma y de su sector público». Boletín Oficial País Vasco,116 ZK, de 19 de junio de 2008). 